\title{
Repercussões da mobilização passiva nas variáveis hemodinâmicas em pacientes sob ventilação mecânica
}

\section{Repercussions of passive mobilization in hemodynamic variables in patients under mechanical ventilation}

\author{
Edwiges Aline Freitas Peixoto Cavalcante ${ }^{1}$, Debora Helen Marques da Silva², David Santos Pontes ${ }^{3}$, Paulo Goberlânio de Barros \\ $\mathrm{Silva}^{4}$, Andréa Stopiglia Guedes Braide ${ }^{4}$, Márcia Cardinalle Correia Viana ${ }^{4,5}$
}

1. Residente em cancerologia pela Escola de Saúde Pública do Ceará (ESP-CE), Fortaleza, CE, Brasil. 2. Pós-graduanda em Ciências Fisiológicas pela Universidade estadual do Ceará (UECE), Fortaleza, CE, Brasil. 3. Fisioterapeuta do Hospital e Maternidade Dra. Zilda Arns, Fortaleza, CE, Brasil. 4. Docente do Curso de Fisioterapia do Centro Universitário Christus (UNICHRISTUS), Fortaleza, CE, Brasil. 5 Fisioterapeuta do Hospital Geral Dr. César Cals (HGCC), Fortaleza, CE, Brasil.

\section{Resumo}

Introdução: A mobilização passiva é amplamente utilizada dentro das unidades de terapia intensiva visando prevenir e/ou minimizar os efeitos deletérios ocasionados pelo imobilismo em pacientes em estado grave. Contudo, pouco se sabe sobre o efeito dessa técnica na hemodinâmica do paciente. Objetivo: Analisar as repercussões da mobilização passiva nas variáveis hemodinâmicas em pacientes sob ventilação mecânica. Métodos: Estudo de campo, observacional, transversal e quantitativo, realizado de agosto de 2015 a maio de 2016. As variáveis de frequência cardíaca (FC); pressão arterial média (PAM), pressão arterial sistólica (PAS), pressão arterial diastólica (PAD) e saturação periférica de oxigênio (Sp02), foram mensuradas em três momentos: antes de iniciar as mobilizações (T1), ao término (T2) e 2 minutos após o término (T3). Resultados: A amostra constou de 15 pacientes, sendo 9 (60\%) do gênero feminino, com idade mediana de 57 anos. Não se constataram alterações significativas nas variáveis FC, PAS e PAM. No entanto, pôde-se notar uma queda significante da PAD ( $\mathrm{p}=0,010)$ e SpO2 $(\mathrm{p}=0,028)$ entre os tempos T2 e T3 na mobilização de membros superiores (MMSS) e alteração significativa da PAS ( $p=0,040)$ na mobilização de MMSS entre os tempos T2 e T3 em pacientes com mais de 60 anos. Conclusão: A realização da mobilização passiva em pacientes sob ventilação mecânica não ocasionou alterações significativas na hemodinâmica do ponto de vista clínico e pode ser considerada uma técnica segura e viável para minimizar os efeitos deletérios gerados pelo imobilismo.

Palavras-chave: Polineuropatias. Variáveis hemodinâmicas. Mobilização passiva. Fisioterapia. Unidades de terapia intensiva.

\begin{abstract}
Introduction: Passive mobilization is widely used within intensive care units to prevent and/or minimize the deleterious effects of immobility in critically ill patients. However, little is known about the effect of this technique on patient's hemodynamics. Objectives: To analyse the repercussions of passive mobilization on hemodynamic variables in patients under mechanical ventilation. Methods: Observational, cross-sectional, and quantitative field study performed from August 2015 to May 2016. The following variables: heart rate (HR); mean blood pressure (MBP); systolic blood pressure (SBP); diastolic blood pressure (DBP) and Peripheral oxygen saturation (Sp02), were measured at three different moments: before initiating the mobilizations (T1), at the end (T2) and two minutes after the end (T3). The collected data were analyzed through the Statistical Package for Social Science (SPSS) software. Results: The sample consisted of 15 patients, nine (60\%) of the female gender and six (40\%) of the male, with a median age of 57 years. There were no significant changes in the HR, SBP and MBP variables. However, a significant decrease in DBP (p = 0.010) and SpO2 (p = 0.028) between T2 and T3 in upper limb mobilization (ULM) and significant SBP change $(p=0.040)$ in mobilization of upper limb between T2 and T3 in patients over 60 years old. Conclusion: Passive mobilization in mechanically ventilated patients did not cause significant changes in hemodynamics from a clinical point of view and can be considered a safe and viable technique to minimize the deleterious effects generated by immobility.
\end{abstract}

Keywords: Polyneuropathies. Hemodynamic variables. Passive mobilization. Physiotherapy. Intensive care units.

\section{INTRODUÇÃO}

Desde a Antiguidade, a prática da Medicina Intensiva consiste, basicamente, no tratamento e na monitorização permanente de doentes graves. As unidades de terapia intensiva (UTI) surgiram com o objetivo de oferecer atenção contínua e suporte diferenciado aos pacientes críticos, com risco de morte, não dispondo, inicialmente, de recursos tecnológicos para assegurar esses cuidados. Com o desenvolvimento da Ciência, houve o surgimento de novos aparatos tecnológicos, que melhoraram a monitorização dos pacientes admitidos nessas unidades e ampliaram sua expectativa de vida ${ }^{1,2}$.
A principal finalidade da UTI é oferecer suporte avançado e atenção contínua para pacientes graves. Essa assistência é realizada por uma equipe multidisciplinar composta por médicos, enfermeiros, fisioterapeutas e terapeutas ocupacionais, com o objetivo de recuperar esses pacientes e minimizar a quantidade de óbitos ${ }^{2}$.

Os pacientes que permanecem por tempo prolongado na UTI tendem a sofrer com os efeitos deletérios da imobilidade, que repercute em contraturas, alterações das fibras musculares,

Correspondência: Márcia Cardinalle Correia Viana. Endereço: Rua João Adolfo Gurgel, 133,Cocó,CEP: 60190-060, Fortaleza, Ceará.E-mail: marciaccorreia@ hotmail.com

Conflito de interesse: Não há conflito de interesse por parte de qualquer um dos autores.

Recebido em: 10 Ago 2017; Revisado em: 26 Dez 2017; 4 Jan 2018; Aceito em: 5 Jan 2018 
fraqueza muscular e atrofia ${ }^{3}$. A associação desses fatores contribui para o aumento do tempo de permanência na unidade, resultando em um maior risco de complicações, aumento na quantidade de óbitos e custos mais elevados ${ }^{4,5}$.

A fraqueza muscular adquirida é considerada uma grave complicação e ocorre principalmente por inflamações sistêmicas, alterações eletrolíticas, deficiências nutricionais, utilização de medicamentos e é agravada pelo repouso ${ }^{6}$. Para Mota e Silva ${ }^{3}$, os efeitos deletérios da imobilização, inclusive a fraqueza muscular generalizada, podem ser prevenidos ou revertidos com a realização da mobilização precoce (MP).

A polineuropatia do paciente crítico é um termo comum; está associada ao tempo prolongado dos pacientes na UTI, aos distúrbios dos vasos e da microcirculação. Apresenta a fraqueza muscular como uma de suas características mais marcantes, caracterizando-se, também, por hiporreflexia que acomete principalmente os grupos musculares distais ${ }^{7}$.

O principal fator para que ocorra essa afecção é uma resposta inflamatória sistêmica, muitas vezes ocasionada pelo mesmo mecanismo da sepse. Ocorrem alterações na microcirculação e seu aparecimento também pode estar relacionado à nutrição e à utilização de fármacos. Cabe ao fisioterapeuta estar atento ao aparecimento dessa doença, para que possa intervir de forma precoce $^{7,8}$.

Tendo em vista que a MP é de competência do fisioterapeuta, é preciso desenvolver o hábito de realizar, de maneira segura, essas mobilizações, embora a viabilidade da realização dessas mobilizações dependa do estado em que se encontra o paciente e se ele tende a ter instabilidade hemodinâmica ou respiratória. Dessa forma, a Fisioterapia vem exercendo um papel importante na UTI, uma vez que proporciona uma melhor recuperação desses pacientes e gera melhora na funcionalidade ${ }^{9,10}$.

Diante do exposto e sabendo-se que a MP é uma técnica bastante utilizada por fisioterapeutas, surgiu o interesse em conhecer quais as possíveis repercussões hemodinâmicas apresentadas pelos pacientes na aplicação da técnica.

Dessa forma, este estudo objetivou analisar as repercussões da mobilização passiva nas variáveis hemodinâmicas em pacientes sob ventilação mecânica (VM) bem como verificar a correlação das alterações encontradas.

\section{MÉTODOS}

Foi realizada uma pesquisa observacional, transversal e quantitativa, no período de agosto de 2015 a maio de 2016. A unidade hospitalar em estudo é referência na atenção terciária à saúde nas áreas de clínica médica, cirurgia, ginecologia, obstetrícia e neonatologia em Fortaleza. Possui duas UTI's para adultos, com seis leitos em cada uma.

Dos 75 pacientes admitidos na UTI no período da coleta, apenas 15 atenderam aos critérios de inclusão do presente estudo. A população foi composta por pacientes com idade acima de 18 anos, de ambos os gêneros, sob VM, sendo a amostra escolhida de forma não probabilística, por conveniência e de acordo com a demanda de pacientes do hospital.

Foram incluídos pacientes sob VM, hemodinamicamente estáveis e em sincronia com o ventilador mecânico. Os pacientes com quadro de broncoespasmo, agitação neuropsicomotora, pressão intracraniana elevada, cavidade abdominal aberta, uso de meias de compressão pneumática, cateter de hemodiálise, rigidez articular, e aqueles com imobilizações de membros por fraturas ou lesões extensas como queimaduras e feridas abertas foram excluídos deste estudo.

Para a coleta de dados, utilizou-se uma ficha de avaliação elaborada pelos pesquisadores, a qual contemplava os objetivos propostos pela pesquisa, sendo a coleta realizada somente após a assinatura do Termo de Consentimento Livre e Esclarecido pelo responsável legal do paciente. Durante o atendimento, os pacientes eram monitorados com o aparelho Dixtal ${ }^{\circledR}$ disposto em cada leito da unidade. As variáveis analisadas foram: FC, $\mathrm{PAS}, \mathrm{PAD}, \mathrm{PAM}$ e SpO2. Além disso, foram coletados dados sobre a idade, o gênero e a patologia de base dos pacientes.

O protocolo da MP de membros superiores (MMSS) consistiu em 15 movimentos de flexão-extensão de punho, seguido por extensão e pronação de cotovelo, 15 movimentos de flexão extensão do ombro com cotovelo estendido, finalizando com 15 movimentos de abdução e adução de ombro com cotovelo estendido.

Para os membros inferiores (MMII), foram realizados 15 movimentos de dorso-flexão e flexão plantar, seguidos por 15 movimentos de flexão e extensão de joelho e quadril, finalizando com 15 movimentos de abdução e adução de quadril.

A mobilização foi realizada durante todo o período do estudo por apenas um dos pesquisadores, sendo a MP de MMSS e MMII executada em tempos divergentes e de acordo com o protocolo estabelecido por este estudo, não sendo cronometrado, porém respeitando a quantidade de repetições definidas.

As variáveis de $\mathrm{FC}$, PAS, $\mathrm{PAD}, \mathrm{PAM}$ e $\mathrm{SpO} 2$ foram mensuradas em três momentos distintos: antes de iniciar as mobilizações (T1), ao término da intervenção (T2) e dois minutos após o término das mobilizações (T3). Respeitou-se o intervalo de um minuto para o início da mobilização do outro membro, quer seja de membro superior ou inferior.

Os dados coletados foram armazenados em um banco de dados com o auxílio do Microsoft Office Excel versão 2010 e, posteriormente, analisados pelo Software estatístico Statistical Package for the Social Science (SPSS) versão 17.0, em que todas as análises foram realizadas com a adoção de uma confiança de $95 \%$.

Em seguida, foram submetidos ao teste de normalidade de Kolmogorov-Smirnov e expressos em forma de média e desvio- 
padrão (dados paramétricos). Para a análise da variação de cada medida nos três momentos, utilizou-se o teste ANOVA para medidas repetidas, seguido do pós-teste de Bonferroni.

Para avaliar a inter-relação entre as variações das medidas de T2 para T3, foi utilizada a correlação de Pearson. E com a finalidade de verificar a influência das características clínicas (sexo, idade $>60$ anos e condição de origem pulmonar ou não), foi utilizada a análise multifatorial ANOVA-3-way seguida do pós-teste de Bonferroni.

A pesquisa seguiu os preceitos da resolução 466/12 do Conselho Nacional de Saúde/Ministério da Saúde11 e foi aprovada pelo Comitê de Ética em Pesquisa (CEP) sob CAAE: 46255015.2.0000.5041.

\section{RESULTADOS}

A amostra consistiu de 15 pacientes, sendo nove (60\%) do gênero feminino. A idade mediana dos pacientes foi 57 anos, sendo a idade mínima 26 e a máxima 82 anos. Os pacientes foram divididos em duas categorias, tendo como referência a proximidade da mediana: até 60 anos de idade $(n=8 ; 53 \%)$ e acima de 60 anos de idade $(n=7 ; 47 \%)$. Em relação aos diagnósticos de base, não se obteve uma prevalência, sendo os mais encontrados: insuficiência cardíaca, pneumonia, doença pulmonar obstrutiva crônica e neoplasia. Eles foram divididos em duas categorias: com associação de doenças cardiorrespiratórias e sem associação de doenças cardiorrespiratórias.

Nenhuma medida relacionada aos exercícios de MMII mostrou variação significante ao longo dos três momentos. Porém, com relação aos MMSS, apesar de não haver diferença significativa na variação de $F C(p=0,338)$, PAS $(p=0,536)$ e PAM $(p=0,158)$, houve queda significante de T2 para T3 da PAD $(p=0,010)$ e da SpO2 ( $p=0,028)$ (Tabela 1$)$.

Com relação aos exercícios realizados nos MMSS, no momento T2 para T3, pôde-se observar que o aumento da FC foi inversamente correlacionado com a redução da $\operatorname{PAD}(p=0,019$, $r=-0,594)$ e que a redução da PAD ocorreu concomitante à redução da PAS ( $p=0,001, r=0,749)$. Consequentemente, a PAM foi diretamente correlacionada à PAS e à $P A D(p<0,001, r=0,932$; $p<0,001, r=0,865$, respectivamente) (Tabela 2).

Tabela 1. Média, desvio-padrão e análise temporal das variáveis hemodinâmicas nos três momentos da MP em MMSS e MMII.

\begin{tabular}{lrrrr}
\hline Variáveis de estudo & T1 & T2 & T3 & p-Valor \\
\hline Membros superiores & & & & \\
FC & $88,2 \pm 15,5$ & $86,0 \pm 13,8$ & $86,5 \pm 15,8$ & \\
PAS & $126,5 \pm 19,8$ & $130,1 \pm 18,2$ & $127,2 \pm 14,9$ & ${ }^{a} 0,338$ \\
PAD & $70,6 \pm 9,7$ & $71,8 \pm 10,5$ & $67,3 \pm 9,1^{*}$ & ${ }^{a} 0,536$ \\
PAM & $89,9 \pm 12,1$ & $91,6 \pm 12,0$ & $87,8 \pm 8,9$ & \\
SpO2 & $95,7 \pm 2,9$ & $96,5 \pm 2,5$ & $95,8 \pm 2,3^{*}$ & \\
Membros inferiores & & & & \\
FC & $86,6 \pm 16,0$ & 86,010 \\
PAS & $126,9 \pm 14,8$ & $86,7 \pm 15,2$ & $85,9 \pm 17,0$ & \\
PAD & $67,9 \pm 8,8$ & $129,9 \pm 17,0$ & $120,9 \pm 20,7$ & \\
PAM & $87,5 \pm 8,8$ & $69,7 \pm 9,5$ & $68,7 \pm 12,8$ & \\
SpO2 & $95,7 \pm 2,5$ & $89,6 \pm 10,3$ & $90,5 \pm 20,4$ & \\
\end{tabular}

${ }^{*} p<0,05$ versus T2, Teste ANOVA para medidas repetidas, seguido do pós-teste de Bonferroni (média $\left.\pm D P\right)$. FC - Frequência cardíaca; PAS - pressão arterial sistólica; PAD- pressão arterial diastólica; PAM- pressão arterial média; SpO2 - saturação periférica de oxigênio.

Tabela 2. Análise de correlação diferença $(\Delta)$ das variáveis hemodinâmicas entre T3 e T2 após MP em MMSS e MMII.

\begin{tabular}{|c|c|c|c|c|c|c|}
\hline \multirow{2}{*}{\multicolumn{2}{|c|}{ Variáveis do estudo }} & \multicolumn{5}{|c|}{ Respectivo Membro } \\
\hline & & $\Delta \mathrm{FC}$ & $\triangle$ PAS & $\triangle$ PAD & $\Delta$ PAM & $\Delta \mathrm{SpO2}$ \\
\hline \multicolumn{7}{|c|}{ Membros superiores } \\
\hline \multirow[t]{2}{*}{$\Delta \mathrm{FC}$} & $\mathrm{R}$ & - & $-0,335$ & $-0,594^{*}$ & $-0,326$ & $-0,462$ \\
\hline & p-Valor & - & 0,222 & 0,019 & 0,236 & 0,083 \\
\hline \multirow[t]{2}{*}{$\triangle$ PAS } & $\mathrm{R}$ & - & - & $0,746^{*}$ & $0,932 *$ & $-0,153$ \\
\hline & p-Valor & - & - & $0,001 *$ & $<0,001^{*}$ & 0,585 \\
\hline \multirow[t]{2}{*}{$\triangle \mathrm{PAD}$} & $\mathrm{R}$ & - & - & - & $0,865^{*}$ & 0,030 \\
\hline & p-Valor & - & - & - & $<0,001^{*}$ & 0,917 \\
\hline
\end{tabular}

J. Health Biol Sci. 2018; 6(2):170-175 


\begin{tabular}{|c|c|c|c|c|c|c|}
\hline \multicolumn{2}{|c|}{ Variáveis do estudo } & \multicolumn{5}{|c|}{ Respectivo Membro } \\
\hline & & $\Delta \mathrm{FC}$ & \multirow{2}{*}{$\begin{array}{r}\Delta \text { PAS } \\
-\end{array}$} & \multirow{2}{*}{$\begin{array}{r}\Delta \text { PAD } \\
-\end{array}$} & \multirow{2}{*}{$\begin{array}{r}\Delta \text { PAM } \\
-\end{array}$} & \multirow{2}{*}{$\begin{array}{r}\Delta \text { SpO2 } \\
-0,145\end{array}$} \\
\hline$\triangle \mathrm{PAM}$ & $\mathrm{R}$ & - & & & & \\
\hline & p-Valor & - & - & - & - & 0,607 \\
\hline \multirow[t]{2}{*}{$\Delta \mathrm{SpO} 2$} & $\mathrm{R}$ & - & - & - & - & - \\
\hline & p-Valor & - & - & - & - & - \\
\hline \multicolumn{7}{|c|}{ Membros inferiores } \\
\hline \multirow[t]{2}{*}{$\Delta \mathrm{FC}$} & $\mathrm{R}$ & - & $-0,317$ & $0,538^{*}$ & $0,519 *$ & 0,409 \\
\hline & $\mathrm{p}$-Valor & - & 0,250 & $0,039 *$ & $0,047^{*}$ & 0,130 \\
\hline \multirow[t]{2}{*}{$\triangle$ PAS } & $\mathrm{R}$ & - & - & $-0,764 *$ & $-0,867^{*}$ & $-0,161$ \\
\hline & $\mathrm{p}$-Valor & - & - & $0,001 *$ & $<0,001^{*}$ & 0,568 \\
\hline \multirow[t]{2}{*}{$\triangle \mathrm{PAD}$} & $\mathrm{R}$ & - & - & - & $0,952 *$ & 0,398 \\
\hline & p-Valor & - & - & - & $<0,001 *$ & 0,142 \\
\hline \multirow[t]{2}{*}{$\triangle$ PAM } & $\mathrm{R}$ & - & - & - & - & 0,331 \\
\hline & p-Valor & - & - & - & - & 0,228 \\
\hline \multirow[t]{2}{*}{$\Delta \mathrm{SpO} 2$} & $\mathrm{R}$ & - & - & - & - & - \\
\hline & p-Valor & - & - & - & - & - \\
\hline
\end{tabular}

${ }^{*} p<0,05$, Correlação de Pearson. $\Delta=$ Variação de T2 para T3.

FC - Frequência cardíaca; PAS - pressão arterial sistólica; PAD- pressão arterial diastólica; PAM- pressão arterial média; SpO2 - saturação periférica de oxigênio.

Nos MMII, no momento T2 para T3, pôde-se observar que a redução da $\mathrm{FC}$ foi diretamente correlacionada com a redução da PAD ( $p=0,039, r=0,538)$, e que a redução da PAD ocorreu concomitante ao aumento da PAS ( $p=0,001, r=-0,764)$, o que fez a PAM ser diretamente correlacionada com a PAD $(p<0,001$, $r=0,952)$ e inversamente com a PAS ( $p<0,001, r=-0,867)$. De T2 para T3, a queda da PAM ocorreu concomitantemente à queda da FC ( $p=0,047, r=0,519)$ (Tabela 2).
Em análise multifatorial, avaliou-se a variação de T2 para T3 e sua relação com as variáveis sexo e idade; pôde-se observar que apenas a PAS mostrou comportamento diferente, considerando a faixa de corte da idade de 60 anos. Pacientes com mais de 60 anos apresentaram aumento significativo da PAS em relação aos pacientes com menos de 60 anos. A variação das demais medidas não foi influenciada significativamente pelo sexo ou pela idade (Tabela 3).

Tabela 3. Valores das médias das variáveis hemodinâmicas em dois tempos distintos durante a MP em MMSS e MMII

\begin{tabular}{lrrr}
\hline Variáveis de estudo & T2-T3 & Sexo & Idade \\
\hline Membros superiores & & & 0,832 \\
FC & $+0,5 \pm 4,4$ & 0,807 & 0,480 \\
PAS & $-2,9 \pm 13,5$ & 0,040 \\
PAD & $-4,5 \pm 4,9$ & 0,863 & 0,615 \\
PAM & $-3,8 \pm 7,3$ & 0,805 & 0,244 \\
SpO2 & $-0,7 \pm 0,8$ & 0,552 & 0,472 \\
Membros inferiores & & & 0,266 \\
FC & $-0,7 \pm 3,2$ & 0,742 \\
PAS & $-9,1 \pm 24,5$ & 0,285 \\
PAD & $-0,9 \pm 8,2$ & 0,635 \\
PAM & $+0,9 \pm 14,3$ & 0,203 \\
SpO2 & $+0,4 \pm 0,7$ & 0,221 \\
\hline
\end{tabular}

* Os pacientes com mais de 60 anos $(+4,7 \pm 5,9)$ apresentaram aumento significativo da PAS de T2 para T3 em relação aos pacientes com menos de 60 anos $(-9,5 \pm 15,0)$. Teste ANOVA-3-way seguido do pós-teste de Bonferroni (média $\pm D P)$.

FC - Frequência cardíaca; PAS - pressão arterial sistólica; PAD- pressão arterial diastólica; PAM- pressão arterial média; SpO2 - saturação periférica de oxigênio. 


\section{DISCUSSÃO}

O objetivo deste estudo foi analisar as repercussões da mobilização passiva nas variáveis hemodinâmicas em pacientes sob VM. Os principais achados foram: a não constatação de alterações hemodinâmicas significativas em nenhuma variável analisada na mobilização de MMII; a redução estatisticamente significativa de T2 para T3 da PAD e da SpO2 na mobilização de MMSS; a correlação inversamente proporcional da FC e PAD nas mobilizações de MMSS e diretamente proporcional nas mobilizações de MMII, no momento T2 para T3; e o aumento significativo da PAS em pacientes com mais de 60 anos. Entretanto, a alteração dos valores dessas variáveis mantiveramse dentro do parâmetro de normalidade clínica, durante todo o período de estudo.

Freitas et al $^{12}$ observaram um aumento significativo da FC com a execução da MP em pacientes sob VM, resultado divergente do que foi encontrado neste estudo. Sabendo-se que o nível de sangue bombeado pelo coração depende das necessidades metabólicas do organismo e que a FC é diretamente proporcional ao débito cardíaco $^{13}$, supõe-se que a distensão e o encurtamento de fibras musculares ocasionadas pela MP do protocolo utilizado no presente estudo não foram suficientes para gerar o aumento deste parâmetro.

Além disso, a FC pode ser interpretada, no contexto geral da condição hemodinâmica do paciente, como limitada pela doença ou pelas reações farmacológicas, fato que pode estar diretamente relacionado aos resultados referentes à FC do presente estudo, de acordo com a variedade de patologias da amostra.

Nossos resultados corroboram os achados de Krzeminski et $\mathrm{al}^{14}$, realizados em indivíduos saudáveis, avaliando respostas cardiovasculares, metabólicas e de catecolaminas circulantes ao exercício ativo e passivo. O autor constatou que o aumento da FC ocorreu apenas no exercício ativo, explicado pela capacidade desse tipo de exercício de promover alterações químicas musculares e estimular a resposta cronotrópica, embora este mecanismo não seja ativado durante a MP.

A pressão arterial é influenciada por diversos fatores, como etnia, estado clínico, sexo, faixa etária, estado de treinamento, tempo de realização, e sofre influência direta de atividades aeróbias realizadas. É caracterizada pela resistência encontrada pelo sangue na parede das artérias como resultado do produto do débito cardíaco e da resistência vascular periférica. Nesse contexto, a PAS, indica a sístole ventricular cardíaca, e a PAD, a diástole ventricular cardíaca; ambas sofrem influência do gasto metabólico ${ }^{15,16}$.

Nesta pesquisa, não se observaram alterações hemodinâmicas significativas referentes à PAM, semelhantes aos achados de Freitas et al12. Isso pode ser explicado pelo fato de que o aumento da resistência vascular periférica e o consequente aumento da pressão arterial são também influenciados pela contração sustentada do músculo ${ }^{17}$. No entanto, uma das características do protocolo foi a frequência cíclica de movimentos mantida e a ausência de contração muscular ativa.

Entretanto, ao analisar a correlação das variáveis hemodinâmicas entre os momentos T2 e T3 após MP em MMSS e MMII, observouse que, nos MMSS, houve um aumento da FC com redução da PAM, enquanto que, em MMII, obteve-se uma redução da FC e da PAM. Apesar de muitas variações encontradas na literatura, sabe-se que exercícios nos membros MMSS ocasionam uma maior sobrecarga cardíaca quando comparados a exercícios de $\mathrm{MMII}^{18}$.

A variável SpO2 manteve-se estável durante os três tempos na MP de MMII. Já na mobilização de MMSS, ocorreu uma redução entre os momentos T2 e T3. Esse achado é divergente do que foi observado por Savi et al $^{19}$ em pacientes submetidos à movimentação cíclica passiva dos MMII que apresentaram aumento do consumo de oxigênio concomitantemente a uma queda na saturação de oxigênio, provavelmente devido a uma elevada taxa de extração de oxigênio e ao índice cardíaco.

Quanto a esse fato, pode-se pressupor que seria necessário um protocolo de mobilizações de MMII com um maior tempo de execução, para que houvesse mais necessidade de captação de oxigênio, o que contribuiria diretamente para uma diminuição da $\mathrm{SpO}_{2}$.

Os pacientes com mais de 60 anos apresentaram aumento significativo da PAS na avalição da variação de T2 para T3 durante a MP de MMSS. Estudos demonstram que o simultâneo alongamento e o encurtamento muscular, que é típico da MP, provocam a ativação dos metabarorreceptores e das fibras tipo III, que podem induzir a inibição vagal e estimular barorreceptores, contribuindo para o aumento da resposta cardiovascular ${ }^{20,21}$.

Segundo Gonzaga et $\mathrm{al}^{22}$, o aumento da deficiência de óxido nítrico encontrado na população idosa resulta em aumento do estresse oxidativo, favorecendo a vasoconstrição, diminuindo, assim, a complacência arterial e a distensibilidade dos vasos, causando aumentos na PAS.

Este estudo tem a limitação de possuir um número de amostra reduzida, o que pode ter contribuído para a ausência de significância em algumas variáveis. No entanto, a intenção foi acrescentar dados à prática clínica do fisioterapeuta em UTI, com a finalidade de assegurar a técnica de MP em pacientes críticos, como estratégia de prevenção e tratamento de complicações ocasionadas pelo imobilismo.

\section{CONCLUSÃO}

De acordo com os resultados obtidos neste estudo, a realização da MP de MMSS e MMII em pacientes sob VM não ocasionou repercussões hemodinâmicas significativas em relação às variáveis FC, PAS e PAM.

Em relação aos MMSS, pôde-se notar uma redução em T2 para 
T3 na PAD e SpO2, além de uma alteração significativa da PAS em pacientes com mais de 60 anos. Essas alterações observadas se mantiveram dentro dos parâmetros de normalidade durante todo o tempo de coleta.

Sugere-se a realização de novas pesquisas com um número maior de pacientes que possam comprovar os achados do presente estudo, visto que a MP em pacientes sob VM é uma terapêutica importante para minimizar os efeitos deletérios gerados pelo imobilismo, sendo necessário monitorização constante durante sua execução.

\section{REFERÊNCIAS}

1 Meneses FA. Iniciação a medicina intensiva: panorâmica do doente adulto. Fortaleza: Faculdade Christus; 2010.

2. Santuzzi CH, Scardua MJ, Reetz JB, Firme KS, Lira NO, Gonçalves WLS. Aspectos éticos e humanizados da fisioterapia na UTI: uma revisão sistemática. Fisioter Mov. 2013; 26(2): 415-22. doi: http://dx.doi.org/10.1590/S010351502013000200019 .

3. Mota CM, Silva VG. A segurança da mobilização precoce em pacientes críticos: uma revisão de literatura. Interfaces Científicas: Saúde e Ambiente. 2012; 1(1): 83-91. doi: http://dx.doi.org/10.17564/2316-3798.2012v1n1p83-91.

4. Basto PAS, Soares YO, Oliveira HS, Gonçalves WS, Balestra LF, Gardenghi G. Repercussões da sedação em pacientes internados em unidades de terapia intensiva: uma revisão sistemática. ASSOBRAFIR Ciência. 2014; 5(2): 59-72.

5. Ultra RB. Unidade de Terapia Intensiva. In: Ultra RB. Fisioterapia intensiva. 4 ed. Rio de Janeiro: Guanabara Koogan; 2009.

6. Silva APP, Maynard K, Cruz MR. Efeitos da fisioterapia motora em pacientes críticos: revisão de literatura. Rev bras ter intensiva. 2010 Mar; 22(1): 85-91. doi: http://dx.doi.org/10.1590/S0103-507X2010000100014.

7. Zamora VEC, Cruz MR. Polineuromiopatia do paciente crítico: uma revisão da literatura. Revista HUPE. 2013; 12(3):118-129. doi:10.12957/rhupe.2013.7539.

8. Zamora VEC, Tachau Joia AIAT, Silva KM. Impacto da polineuromiopatia do paciente crítico no desmame da ventilação mecânica. Fisioter. Bras. 2010 JanFev; 11(1): 54-60.

9. Castro SJ Júnior. A importância da mobilização precoce em pacientes internados na Unidade de Terapia Intensiva (UTI): revisão de literatura. Persp Online: biol. \& saúde. 2013; 03(1):15-23.

10. Feitoza CL, Jesus PKS, Novais RO, Gardenghi G. Eficácia da fisioterapia motora em unidades de terapia intensiva, com ênfase na mobilização precoce. RESC. $2014 ; 4(1)$ : 19-27.

11. Brasil. Ministério da Saúde. Resolução no 466 [Internet], de 12 de dezembro de 2012. Diário Oficial [da] República Federativa Brasil. 2013 Jun 13 [acesso 2015 Jun 15]; Seção 1. p. 59. Disponível em: < http://conselho.saude.gov.br/ resolucoes/2012/Reso466.pdf>.
12. Freitas ERFS, Bersi RSS, Kuromoto MY, Slembarski SC, Sato APA, Carvalho $M Q$. Efeitos da mobilização passiva nas respostas hemodinâmicas agudas em pacientes sob ventilação mecânica. Rev bras ter intensiva. 2012 Jan-Mar; 1(24): 72-78. doi: http://dx.doi.org/10.1590/S0103-507X2012000100011.

13. Preston RR, Wilson TE. Fisiologia ilustrada. Porto Alegre: Artmed; 2014.

14. Krzeminski K, Kruk B, Nazar K, Ziemba W, Cybulsky G, Niewiadomski W. Cardiovascular, metabolic and plasma catecholamine responses to passive and active exercises. J Physiol Pharmacol. 2000 Jun; 51(2):267-78. PubMed PMID: 10898099

15. Polito MD, Farinatte PTV. Respostas de frequência cardíaca, pressão arterial e duplo produto ao exercício contra resistência: uma revisão da literatura. Rev Port Cien Desp. 2003; 3(1):79- 90.

16. Casonato J, Polito MD. Hipotensão pós exercício aeróbio: Uma revisão sistemática. Rev Bras Med Esporte. 2009 Mar-Abr; 15(2):151- 157. doi: http:// dx.doi.org/10.1590/S1517-86922009000200014

17. MacDonald JR, MacDougall JD, Hogben CD. The effects of exercising muscle mass on post exercise hypotension. J Hum Hypertens. 2000 May; 14(5):317-20. PubMed PMID: 10822318.

18. Haddad S. Ergometria de membros superiores: um Método importante na avaliação cardiocirculatória ao exercício. Arq Bras Cardiol. 1997 Set; 69(3):189193. doi: http://dx.doi.org/10.1590/S0066-782X1997000900009.

19. Savi A, Maia CP, Dias AS, Teixeira C. Efeitos hemodinâmicos e metabólicos da mobilização passiva dos membros inferiores em pacientes sob ventilação mecânica. Rev bras ter intensiva. 2010 Out-Dez; 22(4): 315 - 320. doi: http:// dx.doi.org/10.1590/S0103-507X2010000400001.

20. Baum K, Selle K, Leyk D, Essfeld D. Comparison of blood pressure and heart rate responses to isometric exercise and passive muscle stretch in humans. Eur J Appl Physiol Occup Physiol. 1995; 70(3):240-5. PubMed PMID: 7607199.

21. Kaufman MP, Hayes SG. The exercise pressor reflex. Clin Auton Res. 2002 Ded; 12(6):429-39. doi: 10.1007/s10286-002-0059-1.

22. Gonzaga CC, Sousa MG, Amodeo C. Fisiopatologia da hipertensão sistólica isolada. Rev Bras Hipertens. 2009; 16(1):10-14.

Como citar este artigo/How to cite this article:

Cavalcante EAFP, Silva DHM, Pontes DS, Silva PGB, Braide ASG, Viana MCC. Repercussões da mobilização passiva nas variáveis hemodinâmicas em pacientes sob ventilação mecânica. J Health Biol Sci. 2018 Abr-Jun; 6(2):170-175. 